\title{
High-Performance Versatile Setup for Simultaneous Brillouin-Raman Microspectroscopy
}

\author{
F. Scarponi, ${ }^{1}$ S. Mattana, ${ }^{2}$ S. Corezzi, ${ }^{2, *}$ S. Caponi ${ }^{3}$ L. Comez, ${ }^{3}$ P. Sassi ${ }^{4}$ A. Morresi,${ }^{4}$ M. Paolantoni,${ }^{4}$ L. Urbanelli,${ }^{5}$ \\ C. Emiliani, ${ }^{5}$ L. Roscini,${ }^{6}$ L. Corte,${ }^{6}$ G. Cardinali, ${ }^{6}$ F. Palombo, ${ }^{7}$ J. R. Sandercock,,${ }^{1}$ and D. Fioretto ${ }^{2, \dagger}$ \\ ${ }^{1}$ Tablestable Ltd., Im Grindel 6, CH-8932 Mettmenstetten, Switzerland \\ ${ }^{2}$ Dipartimento di Fisica e Geologia, Università di Perugia, Via Pascoli, I-06123 Perugia, Italy \\ ${ }^{3}$ IOM-CNR, clo Dipartimento di Fisica e Geologia, Università di Perugia, \\ Via Pascoli, I-06123 Perugia, Italy \\ ${ }^{4}$ Dipartimento di Chimica, Biologia e Biotecnologie, Università di Perugia, \\ Via Elce di Sotto 8, I-06123 Perugia, Italy \\ ${ }^{5}$ Dipartimento di Chimica, Biologia e Biotecnologie, Università di Perugia, \\ Via del Giochetto, I-06123 Perugia, Italy \\ ${ }^{6}$ Department of Pharmaceutical Sciences-Microbiology, University of Perugia, \\ Borgo XX Giugno 74, 06121 Perugia, Italy \\ ${ }^{7}$ University of Exeter, School of Physics and Astronomy, Exeter EX4 4QL, United Kingdom \\ (Received 21 February 2017; revised manuscript received 8 June 2017; published 21 July 2017)
}

\begin{abstract}
Brillouin and Raman scattering spectroscopy are established techniques for the nondestructive contactless and label-free readout of mechanical, chemical, and structural properties of condensed matter. Brillouin-Raman investigations currently require separate measurements and a site-matched approach to obtain complementary information from a sample. Here, we demonstrate a new concept of fully scanning multimodal microspectroscopy for simultaneous detection of Brillouin and Raman light scattering in an exceptionally wide spectral range, from fractions of $\mathrm{GHz}$ to hundreds of $\mathrm{THz}$. It yields an unprecedented $150-\mathrm{dB}$ contrast, which is especially important for the analysis of opaque or turbid media such as biomedical samples, and spatial resolution on a subcellular scale. We report the first applications of this new multimodal method to a range of systems, from a single cell to the fast reaction kinetics of a curing process, and the mechanochemical mapping of highly scattering biological samples.
\end{abstract}

DOI: 10.1103/PhysRevX.7.031015

Brillouin and Raman spectroscopy are inelastic light scattering techniques that, by differing only in the probed frequency range, give complementary information on mechanical and chemical properties of matter. Despite their origin being in the same years (1920s) [1,2], the two techniques have been developed as separate tools, also in combination with optical microscopy. Brillouin light scattering (BLS) is the inelastic scattering of light from collective modes, such as acoustic waves (phonons) and spin waves (magnons) propagating in condensed matter, that induce frequency shifts of the radiation in the range $0.1-100 \mathrm{GHz}$. BLS spectroscopy, providing information on the elastic [3,4], viscoelastic [5], and magnetic properties of matter [6,7], has been widely applied in condensed matter physics, including

\footnotetext{
*Corresponding author. silvia.corezzi@unipg.it

Corresponding author. daniele.fioretto@unipg.it

Published by the American Physical Society under the terms of the Creative Commons Attribution 4.0 International license. Further distribution of this work must maintain attribution to the author(s) and the published article's title, journal citation, and DOI.
}

Subject Areas: Interdisciplinary Physics,

Optics, Soft Matter investigations of fibers $[3,8]$, nanoparticles $[9,10]$, and structured materials $[4,11]$, but it has only sparingly been used in the biomedical field [12-15], where it can provide a high-frequency counterpart to traditional mechanical techniques [16]. Raman scattering (RS) involves light inelastically scattered by optical phonons or intramolecular modes, with frequency shifts typically larger than $1 \mathrm{THz}$. An RS spectrum is a truly chemical fingerprint of the material with information concerning the molecular composition and structure. RS is widely used in a number of fields ranging from analytical and physical chemistry to biophotonics and biomedical sciences [17]. Recently, the conventional barrier between the two techniques has been crumbling and increasing interest is devoted to simultaneous Brillouin and Raman light scattering investigations. Extended depolarized light scattering (EDLS) based on separate Brillouin and Raman measurements has been introduced to study solvation processes [18] and complex relaxation patterns in glasses [19] and glass-forming materials [20]. Also, Brillouin and Raman microscopy have been applied through a site-matched approach to obtain mechanical mapping with chemical specificity of human tissues $[21,22]$, thus opening the route to a wide range of biomedical and bioengineering applications. Although the contactless nature of light scattering 
considerably improves the potential for noninvasive realtime, in vivo applications, the full development of Brillouin microscopy has been hampered by long acquisition times [of the order of minutes with traditional Fabry-Pérot (FP) interferometers].

Advances in Brillouin microscopy have recently been achieved by introducing a nonscanning virtually imaged phase array (VIPA) in place of the Fabry-Pérot interferometer $[14,15,23]$. The low contrast of VIPA has been improved by multipass configurations [24], eventually used in combination with a triple-pass Fabry-Pérot interferometer as a bandpass filter [25] or a molecular or atomic gas cell as a notch filter [26]. The latter solution has also been adopted in a joint BLS-RS setup [27,28], although with improvements on spectral response (especially Raman) still to be implemented. While VIPAs considerably improve the Brillouin data gathering speed, this comes at a cost: (i) a rather coarse spectral resolution, which is limited to $\sim 0.7 \mathrm{GHz}$ by the fixed thickness of the etalon; (ii) a low contrast, which is $30 \mathrm{~dB}$ in the single-pass setup and reaches $85 \mathrm{~dB}$ in the filter-combined multipass configuration; (iii) a reduced spectral range, which is limited to some tens of $\mathrm{GHz}$ by the repetition of orders in the etalon response function. The VIPA-based approach remains limited to transparent or moderately turbid media, e.g., tissue phantoms, because available spectrometers have not yet achieved the contrast that is required to interrogate truly opaque media such as most biological samples. Furthermore, a better resolution of the spectrometer is needed to accurately measure the linewidth of Brillouin peaks, and to migrate from a purely mechanical characterization (related only to the peak frequency) towards a viscoelastic characterization (related to the peak frequency and linewidth) of biomaterials, with the possibility to distinguish between heterogeneous and homogeneous broadening of Brillouin lines. Therefore, in those cases that require a detailed spectral analysis, a scanning FP-based Brillouin system is the preferable method. On the Raman side, recent developments in nonlinear techniques, e.g., coherent anti-Stokes Raman scattering and stimulated Raman scattering, provide rapid optical sectioning for label-free imaging of biomaterials with limited depth of penetration. However, the reduced spectral resolution and the presence of a nonresonant background still limit the sensitivity and hamper the applications of coherent anti-Stokes Raman scattering; stimulated Raman scattering seems more promising, provided new laser systems for the "fingerprint" region will become available.

In this article, we present a high-resolution, high-contrast, and wide spectral range confocal microspectroscopic setup based on a new concept of multipass FP interferometer and a conventional Raman spectrometer, enabling simultaneous Brillouin-Raman microspectroscopy (BRMS) for a range of applications unapproachable by ordinary devices.

Figure 1(a) shows a schematic of the setup. Visible light from a laser source $(S)$ is focused onto a sample by the same objective that is also used to collect the backscattered light. The sample is mounted onto a three-axes piezotranslation stage ( $\mathrm{SH})$ for mapping measurements. A polarizing beam splitter (BS) transmits the depolarized backscattered light through to the spectrometers. Immediately after, a short-pass tunable edge filter (TEF) transmits the quasielastic scattered light to a new concept of tandem FabryPérot interferometer (TFP-2 HC; see Methods) and reflects the deeply inelastic scattered light into a Raman monochromator (RM).

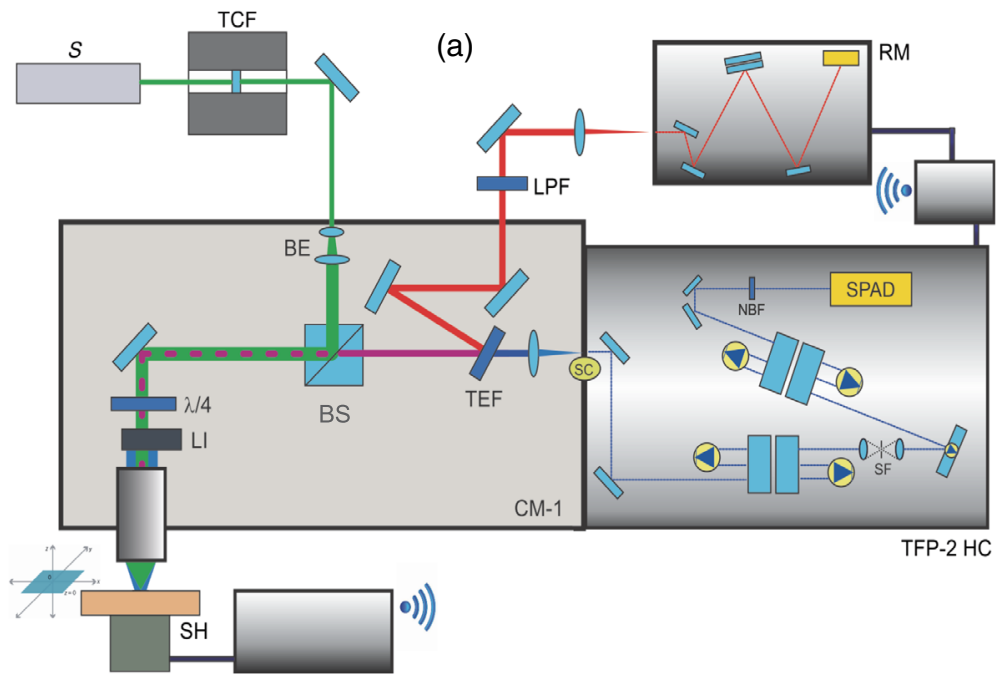

(b)

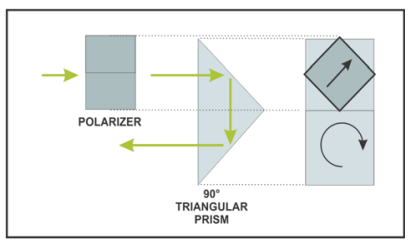

(c)

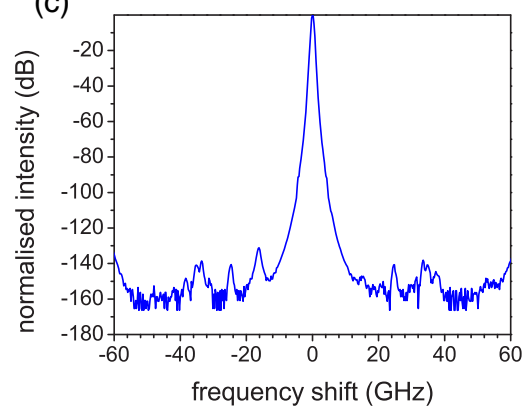

FIG. 1 (a) Schematic of the Brillouin-Raman microspectroscopy (BRMS) setup, consisting of a confocal microscope (CM-1), a tandem Fabry-Pérot interferometer (TFP-2 HC), and a Raman monochromator (RM) (see Methods for more details). (b) Scheme of the optical isolators used in the TFP-2 HC (side and front view) also represented by the small diodelike symbols in (a). The instrument uses circular polarizing techniques to prevent back reflections and so completely decouple the separate passes. (c) Spectral response of the TFP-2 HC interferometer. This yields an instrumental contrast better than $150 \mathrm{~dB}$. 
The tandem Fabry-Pérot interferometer, which we present here for the first time, reaches the unprecedented $>150-\mathrm{dB}$ contrast in just a $3+3$ pass configuration thanks to the use of optical isolators, but also preserves a high luminosity through the use of an avalanche-photodiode detector. This configuration enables one to exploit all the scattered light, to improve by more than $50 \mathrm{~dB}$ the contrast with respect to traditional $3+3$ pass interferometers, making it possible to measure truly opaque samples and, eventually, to reduce the acquisition time to values typical of Raman spectrometers. This enables joint Brillouin-Raman rapid raster-scan mapping and time-sampling experiments.

To demonstrate the performances of the new instrument, here we report the results of Brillouin-Raman mapping of a microbial biofilm. Biofilms are ubiquitous structures [29] formed by microbial cells growing onto solid surfaces embedded in a polymer matrix, often made of esopolysaccharides, produced by the cells themselves [30]. City of microbes [31] with a complex morphology, biofilms are important because of their increased resistance to antibiotics, antifungal drugs, and extreme conditions $[5,32,33]$. Despite being poorly understood, the mechanical characteristics of the biofilm are of primary interest to elucidate the mechanisms governing the stability and the dispersion of cells within the biofilm [34,35].
Figure 2(a) shows a photomicrograph of a Candida albicans biofilm in which a multilayer cluster of roundshaped cells is visible. The Brillouin and Raman maps of the sample are obtained using a $50 \times$ objective, $1-\mu \mathrm{m}$ stepsize, $20 \times 20$ points. Spectra are collected simultaneously, with an acquisition time of $10 \mathrm{~s}$ and an incident laser power of $17 \mathrm{~mW}$. Figure 2(d) shows that a contrast higher than $110 \mathrm{~dB}$ is required to detect longitudinal phonons in this highly scattering sample. Raman maps are obtained by plotting the intensity (integral) of the bands in the range $2800-3000 \mathrm{~cm}^{-1}$ [Fig. 2(b)], showing the prominent $\mathrm{CH}_{2}$ stretching mode of proteins and lipids, and $1200-1530 \mathrm{~cm}^{-1}$ [Fig. 2(c)], the socalled fingerprint region, comprising bands due to the amide III, cytochrome c, carbohydrates, proteins, and lipids. The observed heterogeneity in the concentration of $\mathrm{CH}$ groups in molecules across the scanned area can be attributed to a nonuniform thickness of the biofilm, with an increase in intensity by a factor of $2-3$ in the transition region from one to two or more Candida layers. The most interesting feature is the high intensity of Raman bands in the central part of the biofilm. In this region, a prominent maximum in the bands at $1200-1530 \mathrm{~cm}^{-1}$, whose intensity increases by a factor 20 , can not only be attributed to the multilayer structure of the biofilm, but also to a modification in the cell status and/or in the biofilm composition. In particular, we can expect the

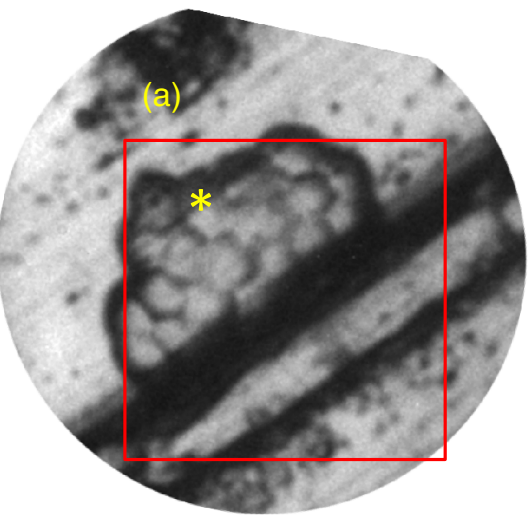

(d)

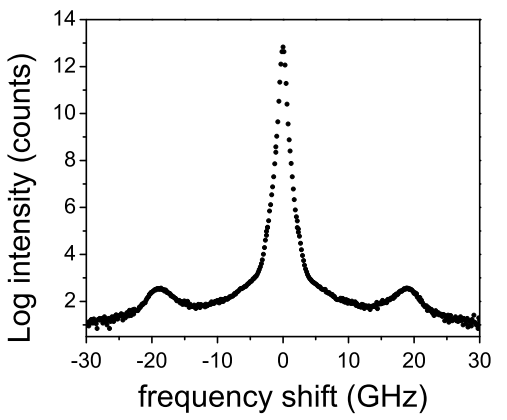

(b)

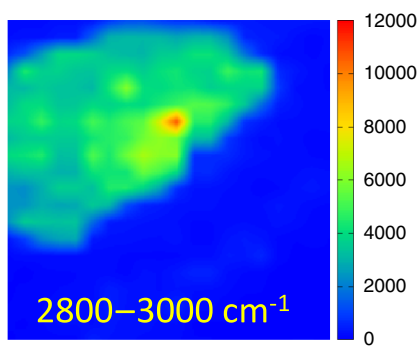

(e)

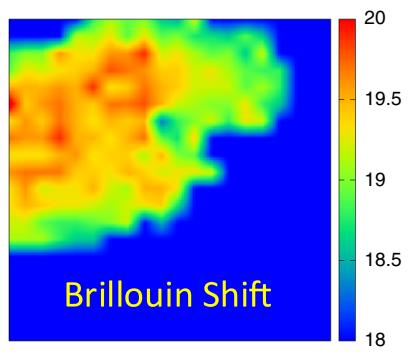

(c)

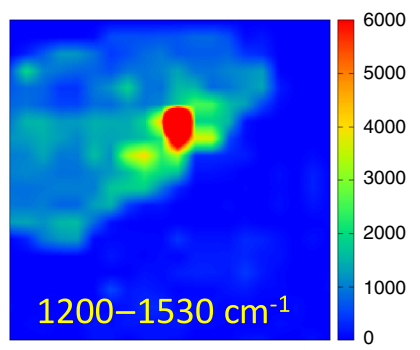

(f)

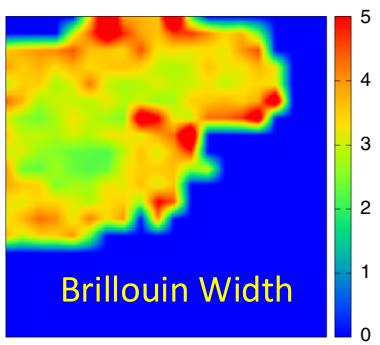

FIG. 2. (a) Optical micrograph of a Candida albicans biofilm. The red box denotes the $20 \times 20 \mu \mathrm{m}^{2}$ area whereby Brillouin-Raman raster-scan maps are obtained. (b),(c) Raman map based on the integrated intensity of the bands in the region indicated in the label. (d) Brillouin spectrum extracted from the point denoted by an asterisk in (a), showing that a minimum contrast of approximately $110 \mathrm{~dB}$ is required to detect longitudinal phonons in this sample. The quasielastic signal $(<2 \mathrm{GHz})$ is acquired through the same procedure as that used to obtain the spectrum in Fig. 1(c) (see Methods), i.e., with the laser beam attenuated by calibrated neutral density filters. (e),(f) Brillouin map based on the characteristic frequency $\nu(\mathrm{GHz})$ and linewidth $\Gamma(\mathrm{GHz})$ of the Brillouin peak. 
presence of zones where more extracellular polymeric substance is present and where the survival of Candida cells is favored. In this respect, it is interesting to note that the marked (out-of-scale) increase of Raman signals in the range $1200-1530 \mathrm{~cm}^{-1}$ could be at least partially attributed to the resonant scattering of cytocrome $\mathrm{c}$, a marker of cell vitality $[36,37]$. Crucial to understanding this feature is the noticeable change in the viscoelastic properties of the biofilm, visible in the Brillouin maps [Figs. 2(e) and 2(f)]. An elasticity map is inferred from the frequency map in Fig. 2(e) under the assumptions that the detected phonons are acoustic with linear dispersive behavior, and that the ratio $\rho / n^{2}$ - with $\rho$ the density of the sample and $n$ its refractive index - is approximately constant through the scanned area (see Methods). Greater care in the Brillouin spectrum interpretation is necessary when these conditions are not satisfied, e.g., in microstructured materials with strong mechanical nonlinearity [4] or in mesoscopic structures such as opals and phononic crystals [4,11]. Refractive index and density are not uniform throughout the sample; however, $\rho / n^{2}$ is constant with good approximation far from electronic resonances, so that the relative variation of the frequency squared $\nu^{2}$ gives a good estimation of the variation of the elastic modulus. The actual changes in refractive index and density typically amount to less than $2 \%$ uncertainty in the elastic modulus estimation $[15,23,38]$, and therefore do not affect the biomechanical characterization of the sample. In the central region of the biofilm, a $\sim 6 \%$ reduction of the Brillouin frequency corresponding to a $\sim 12 \%$ reduction in stiffness of the sample is observed, together with a twofold increase in linewidth (related to the acoustic attenuation in the sample). We note that the presence of multiple scattering in the Brillouin spectra of the biofilm, which is typical of opaque scattering media, does not substantially affect the linewidth interpretation. The combination of reduced stiffness and increased acoustic attenuation has already been observed in dried biological tissues and attributed to the plasticizing effect associated with an increase of residual water [21]. In fact, both spectral changes we observe here can be attributed to a reduction of the structural relaxation time, i.e., of local viscosity, associated to a local increase in hydration level. Note that, in the absence of independent information on the local viscosity of the sample derived from the linewidth of Brillouin peaks, it is not possible to distinguish between the true observed relaxational effect and an alternative fallacious interpretation in terms of a merely elastic effect related to, e.g., a reduction in microfilm connectivity. At the same time, without foundational molecular insight provided by simultaneous Raman spectra, it is not possible to adequately interpret the observed micromechanical properties, which makes the fully integrated Brillouin-Raman setup needed and necessary.

The observed physicochemical heterogeneity (sample thickness, viscoelastic behavior, and chemical modification) is compatible with the presence of a region with live
Candida cells. This result is in line with the microbiological evidence that the biofilm acts as a structure that increases the resistance of cells to challenging agents. In fact, it is plausible that, in the region of larger thickness, the buried cells are protected by the overlaying biofilm structure. The protecting layer is also able to preserve water inside the inner cells, as suggested by the "softer" behavior revealed by Brillouin scattering.

A second experiment shows the potential of BRMS measurements to monitor fast mechanical and chemical changes occurring in reacting samples. The production process of technologically important materials is often multistage [39] or involves complex thermal cycles of reaction. Here, one major need for constructing mechanically optimized products is the synchronized determination of mechanical properties and extent of reaction throughout the process. To test the capability of our system, Fig. 3 shows the time evolution of Brillouin and Raman spectra during the isothermal polymerization of an epoxybased thermoset resin made of diglycidyl ether of bisphenol A (DGEBA) and diethylenetriamine (DETA), which is used as a matrix in high-performance composites [40].

Brillouin spectra [Fig. 3(a)] are taken every $0.5 \mathrm{~s}$, with $30-\mathrm{mW}$ laser intensity. The hardening of the resin is indicated by a progressive increase of frequency $\nu$ and a parallel decrease of linewidth $\Gamma$ of the Brillouin peaks [Fig. 3(c)]; these parameters are related to the longitudinal elastic modulus and longitudinal viscosity, respectively (see Methods). Indeed, the hardening of the resin results from the combination of two major effects, an increase of elastic energy density due to the formation of chemical bonds between DGEBA and DETA molecules and an increase in structural relaxation time of the system as it approaches the glass transition.

The molecular parameter we use to monitor the advancement of the reaction is the conversion $\alpha$, i.e., the fraction of reacted epoxy rings during the curing process. Raman spectroscopy can directly access this parameter through the normalized intensity $I(t)=I_{1255}(t) / I_{1610}(t)$, where $I_{1255}(t)$ is the intensity of the epoxy band at $1255 \mathrm{~cm}^{-1}$ and $I_{1610}(t)$ is the intensity of the phenyl ring stretching band at $1610 \mathrm{~cm}^{-1}$ [41]. In fact, the epoxy groups are consumed during the reaction, while phenyl rings are unaffected by the curing process. The time evolution of the conversion, measured as $\alpha(t)=1-I(t) / I(0)$, is reported in Fig. 3(d) together with the result of a polynomial fit. A transition is observed in the evolution of $\nu^{2}$ versus $\alpha$ at $8 \mathrm{~min}$ from the beginning, i.e., at about $\alpha=0.49$ [Fig. 3(e)]. This decrease in the rate of change of the sound velocity occurs in the reaction range where the sol-gel transition is expected $\left(\alpha_{\text {gel }}=0.5\right.$ according to the FloryStockmayer theory [42]) and the system also goes through the glass transition. In the absence of relaxation processes, $\nu^{2}$ is proportional to the elastic modulus (see Methods) that, in turn, is proportional to the energy density of chemical 

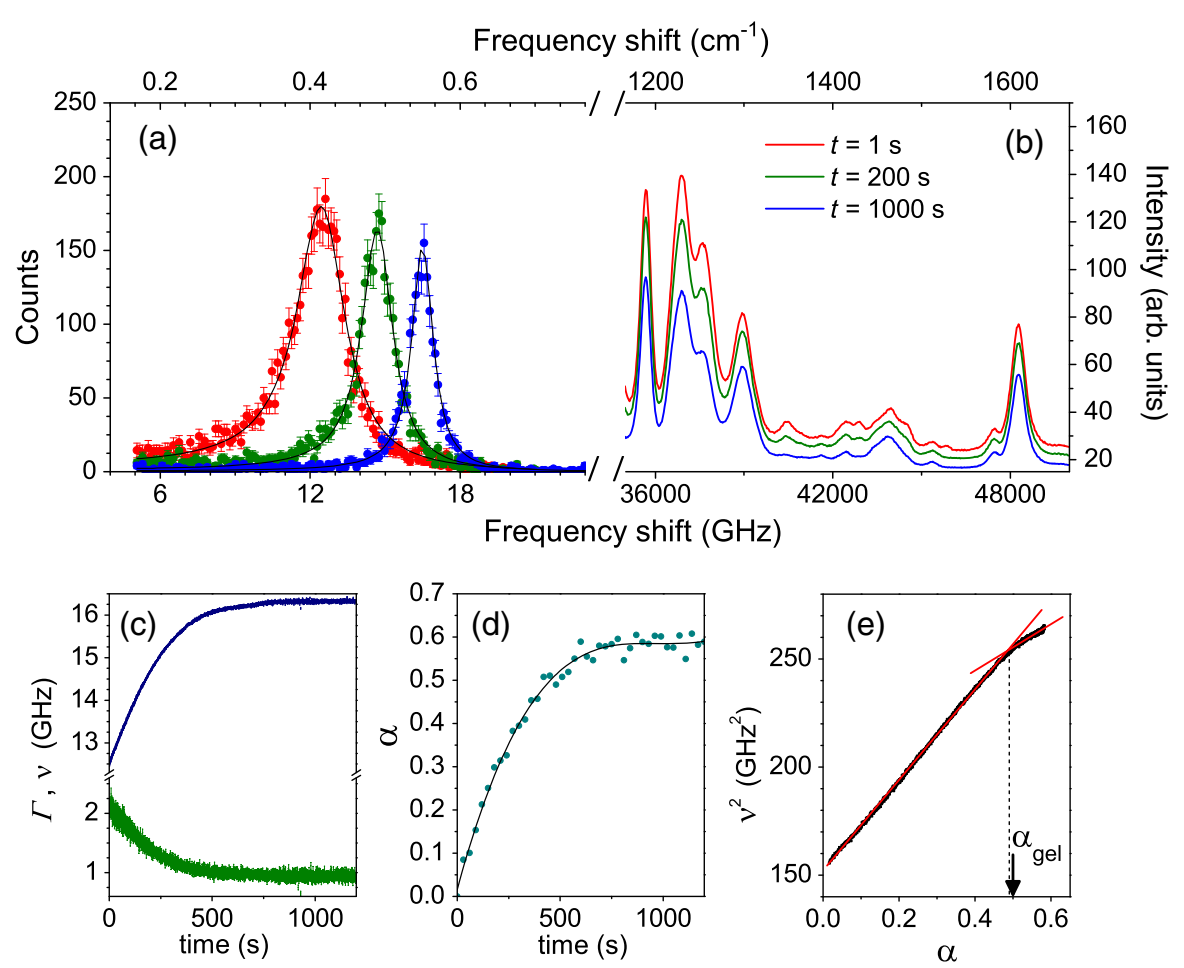

FIG. 3 (a) Brillouin and (b) Raman spectra of an epoxy-amine mixture (DGEBA-DETA 5:2) measured at different times of the isothermal polymerization process, at $T=65^{\circ} \mathrm{C}$ : red symbols, $1 \mathrm{~s}$; green symbols, $200 \mathrm{~s}$; blue symbol, $1000 \mathrm{~s}$ since the beginning. Lines are the results of curve-fit analysis applied to the Brillouin peaks (see Methods). (c) Temporal evolution of the characteristic frequency $(\nu)$ and linewidth $(\Gamma)$ of Brillouin peaks. (d) Time evolution of the conversion $\alpha$ calculated from the intensity of Raman peaks (see text).

(e) Plot of the Brillouin frequency squared versus $\alpha$. The sol-gel transition is expected to occur at $\alpha_{\text {gel }}=0.5$.

bonds, i.e., to the number of bonds formed during the reaction. This condition is better satisfied in the second part of the reaction, and can explain the linear behavior of $\nu^{2}$ versus $\alpha$ for $\alpha>0.49$. Conversely, the presence of the structural relaxation and its evolution during the reaction [43], attested by the variation of $\Gamma$ in Fig. 3(c), is possibly responsible for the faster variation of $\nu^{2}$ versus $\alpha$ in the first part of the reaction. How the complex interplay between structural relaxation and bond formation can generate the linear behavior of $\nu^{2}$ versus $\alpha$ for $\alpha<0.49$ is still unclear. If this is found to be general, the frequency of Brillouin peaks could be used as an effective probe for the advancement of a polymerization reaction.

While the separate monitoring of polymerization processes through Raman [44] and Brillouin scattering goes back a long way $[45,46]$, to the best of our knowledge, this is the first simultaneous measurement of chemical and mechanical evolution during a polymerization reaction.

A third experiment benefits from the wide tunable spectral range accessible to the tandem Fabry-Pérot interferometer and its polarization selectivity to perform extended depolarized light scattering investigations of biological matter.

EDLS spectroscopy has been recently developed to access both vibrational and relaxation dynamics of matter in the wide frequency range between fractions of $\mathrm{GHz}$ and tens of $\mathrm{THz}$ [18]. In this range, it is possible to deal with various topics of condensed matter, going from hydration of biological systems [47] to anharmonicity and boson peak in glasses [48] and highly viscous media [19,49]. EDLS measurements, until now carried out by means of separate dispersive and interferometric setups, can now be performed with a unique instrument, simultaneously, and with high spatial resolution, paving the way to the development of micro-EDLS.

Figure 4 shows the susceptibility representation of the EDLS spectrum of a NIH/3T3 murine fibroblast, measured in the central region of the nucleus. The solvent-free spectrum obtained after the subtraction of the solvent contribution is also reported, together with the spectrum previously obtained for an aqueous solution of a protein using a conventional setup [50]. The spectrum from the nucleus of the cell is dominated by a strong vibrational contribution around $1 \mathrm{THz}$. In addition to possible local vibrations in the complex structure of the nucleus, this contribution can be associated to the boson peak [51], a ubiquitous signature of collective vibrations in disordered condensed matter [52]. Conversely, the strong contribution at $2-3 \mathrm{THz}$ in the spectrum of the protein is mainly attributed to the libration of methyl groups [53]. The number of methyl groups in DNA is much smaller than in proteins [54], and this can explain the suppression of the $2-\mathrm{THz}$ contribution in the spectrum of the nucleus. Worthy 


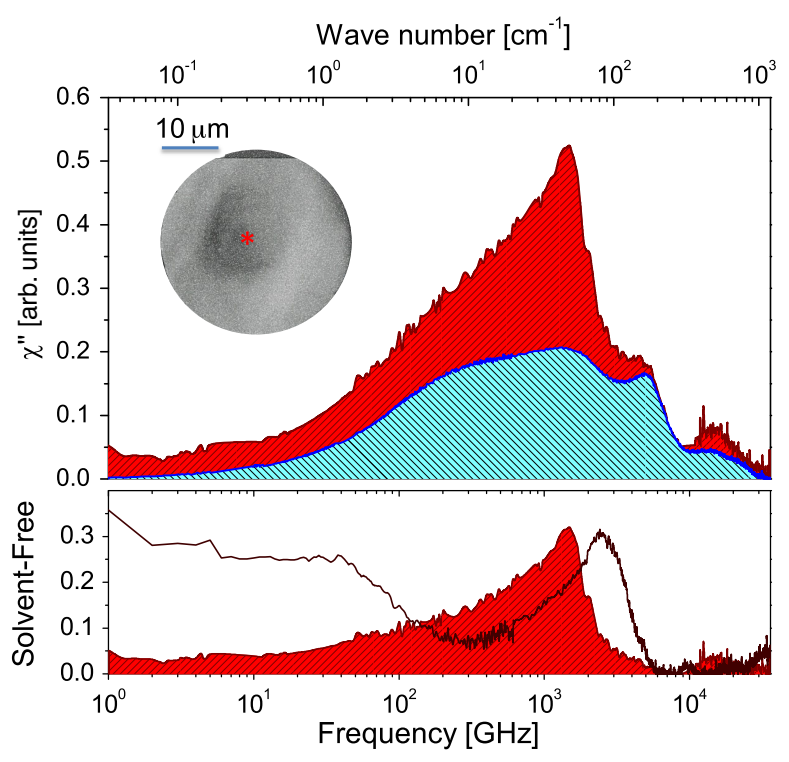

FIG. 4 Top: EDLS susceptibility of a fixed NIH/3T3 fibroblast cell line (red profile) and PBS solution (cyan profile), at $T=25^{\circ} \mathrm{C}$. Each broadband spectral profile has been obtained merging five interferometric spectra and one Raman spectrum in distinct measurements. Inset: Image of a NIH/3T3 single cell acquired with a 50× Mitutoyo objective. Bottom: Solvent-free spectrum obtained by subtracting the spectral profile of the PBS solvent from that of the cell (red profile). The solvent-free spectrum of a $100-\mathrm{mg} / \mathrm{ml}$ lysozyme aqueous solution [47] is also shown for comparison (brown line).

of note is also the $\mathrm{GHz}$ range, where the spectrum is noticeably less intense than in the water-protein solution. In fact, the relaxation processes in DNA are known to be about 1 order of magnitude slower than in proteins [54]; hence, their spectral signature is out of this spectral range. The residual intensity in the $10-100-\mathrm{GHz}$ region can be attributed to the relaxation of hydration water, which is dynamically retarded by a factor greater than 3 with respect to bulk water.

To our knowledge, BRMS gives the first evidence of boson peak and relaxational contributions from hydration water within the nucleus of a single cell. These picosecond motions have been proposed to be responsible for mediating biochemical reactions and energy transport in biological matter $[55,56]$ and for controlling drug intercalation in DNA [57].

In conclusion, we demonstrate rapid and simultaneous Brillouin and Raman experiments, with high contrast, high resolution, and extended spectral range. The capabilities of the novel technique are tested in three case studies: (i) the in situ raster-scan mapping of viscoelastic and chemical properties of biofilms, with the potential of revealing "persister" cells, i.e., cells that are able to survive in the presence of devitalizing agents and are amongst the major causes of hospital infections; (ii) the simultaneous monitoring of mechanical and chemical changes in reacting materials with subsecond time resolution (and fractions of picoliter sample volumes); (iii) the collective dynamics of biological matter by extended depolarized light scattering with subcellular spatial resolution. This noninvasive method is now available for a variety of applications, opening the route for in situ investigation of diverse phenomena in material sciences and potentially for in vivo diagnosis of pathologies involving mechanical changes as well as altered structure and composition.

Methods: Experimental setup.-Figure 1(a) shows a schematic of the experimental arrangement for BrillouinRaman measurements. A 532-nm single-mode solid-state laser (SpectraPhysics Excelsior) is employed as the light source $(S)$. The vertically polarized laser beam is first sent through a JRS Scientific Instruments TCF-1 temperaturecontrolled etalon, which reduces the intensity of laser sidelobes by a factor of about 600 , and then to the input of a customized JRS Scientific Instruments CM-1 confocal microscope, specifically developed in collaboration with the manufacturer to allow Raman-Brillouin signal splitting.

Inside the microscope, the laser beam is first expanded and collimated (BE), then a broadband polarizing beam splitter (BS) sends it to an infinity-corrected apochromatic microscopic objective. In the present experiments, we use a Mitutoyo M-Plan Apo $20 \times$ with a very long working distance of $20 \mathrm{~mm}$, a 2- $\mu \mathrm{m}$ depth of field, and a limiting numerical aperture of 0.42, and a Mitutoyo M-Plan Apo $50 \times$ with working distance of $13 \mathrm{~mm}$ and numerical aperture of 0.55 . The objective is used both to focus the laser beam onto the sample and to collect the backscattered light. The laser spot diameter on the surface is $\sim 2 \mu \mathrm{m}$ measured with the $20 \times$ objective. Illumination of the sample surface is obtained by means of a coaxial LED illuminator (LI), providing blue light peaked at about $470 \mathrm{~nm}$. A broadband $\lambda / 4$ retarder wave plate can be inserted upstream of the objective to switch from depolarized to unpolarized scattering configuration.

The polarizing beam splitter forwards the depolarized backscattered light towards the spectrometers. In the collimated beam section of the microscope, a tunable ultrasteep short-pass filter (TEF, Semrock SP01-561RU) transmits the anti-Stokes quasielastic scattered light to a JRS Scientific Instruments TFP-2 HC Fabry-Pérot interferometer and reflects the Stokes deeply inelastic scattered light towards the Horiba iHR320 Triax Raman spectrometer (RM). The Fabry-Pérot spectrometer includes a narrow band pass filter (NBF) to rule out broadband light signals, while a further RazorEdge long-pass filter (LPF) improves the rejection of residual elastic contributions on the Raman signal line.

Given the different cross section for Raman and Brillouin phenomena, Brillouin spectrometers are usually equipped with the most sensitive detectors, namely, photomultipliers operating in the single-photon regime. In recent times, technologic improvements have led to the development of single-photon avalanche photodiodes (SPAD), reaching 
much higher quantum efficiencies than conventional phototubes. In our setup, a LaserComponents COUNT®-10 SPAD detector is used as a sensor inside the TFP-2 HC spectrometer. This device provides a quantum efficiency of $\sim 70 \%$ at $532 \mathrm{~nm}$, together with a maximum noise rate of $10 \mathrm{cps}$. The achievable signal-to-noise ratio is comparable with that of classical phototube detectors, with an approximate fivefold gain in terms of data-gathering speed.

Pin holes at the entrance of the TFP spectrometer provide a confocal arrangement for microscopic imaging: the broadband light gathered by the objective is imaged onto a USB CMOS sensor camera (SC), which allows us to visualize the sample surface. The field-of-view diameter depends on the objective focal length: using the $20 \times$ objective, it is $\sim 87 \mu \mathrm{m}$. The use of both a polarizer beam splitter and a laser line OD6 notch filter before the camera enables the image to be seen with no loss of signal during the joint measurements.

For mapping measurements, the sample is mounted onto a PI 611-3S Nanocube XYZ closed-loop piezoelectric translation stage $(\mathrm{SH})$, with resolution of $1 \mathrm{~nm}$ and a motion range of $100 \mu \mathrm{m}$ per axis. The high voltage needed by the positioner is generated by a PI E-664 controller, voltage controlled through a homemade DAC/802.11 wireless control board. A software scripting system was developed starting from the JRS automated measurement application in order to execute sequences of simultaneous Raman-Brillouin measurements and stage positioning operations. LabSpec 5 software is used for acquisition of the Raman data and JRS GHOST 7 for the Brillouin data.

The TFP-2 HC is a very high contrast variant of the original Sandercock-type tandem multipass interferometer [58]. The tandem configuration considerably increases the spectral range available for Brillouin and depolarized light scattering experiments, through the suppression of replicas of inelastic signals due to the side orders. In the high-contrast model, polarization and retardation optics [Fig. 1(b)] are used at each pass of the interferometer to effectively decouple each pass from the next one, and a spatial filter (SF) is used to optically decouple the first Fabry-Pérot interferometer from the second one, improving the extinction by a factor higher than $10^{5}$. Before each passage through a Fabry-Pérot interferometer, light passes through a $45^{\circ}$ polarizer and is back reflected with circular polarization by a triangular prism. Light reflected from the next optical surface has opposite chirality and emerges at the polarizer with crossed polarization and is stopped. The adoption of this method also allows the manufacturer to use a perfectly orthogonal incidence of light on all surfaces, thus aiming at the theoretical contrast limit of $10^{18}$.

The laser spectrum in Fig. 1(c) is measured normalizing and merging several spectra of a laser beam at different values of the input power. During these measurements, the sidelobes of the laser spectrum are attenuated by means of a series of two JRS TCF-1 temperature-controlled etalons, with a free spectral range (FSR) of about $68 \mathrm{GHz}$, both tuned to the laser frequency. The mirror spacing of the spectrometer is set at $2.20 \mathrm{~mm}$ in order to match the FSR of the etalon, and small input and output pinholes are used to reduce contributions from uncollimated light. The output power of the laser beam $(\sim 70 \mathrm{~mW})$ is attenuated by means of a set of neutral density filters and a variable one.

Further investigations are in progress to understand the origin of the residual features visible in Fig. 1(c) at the $10^{-15}$ level. The tiny inelastic structures may be attributed to residual sidelobes of the original laser spectrum and also to spurious Brillouin scattering from optical components in the first part of the spectrometer. We can thus anticipate that the true contrast of the interferometer is greater than $150 \mathrm{~dB}$.

Moreover, it has to be noticed that the contrast [Fig. 1(c)] is measured for vertically polarized light, because of the polarization selectivity of the instrument. Supposing to change the polarization input, as for the effect of depolarized elastic scattering, the five polarizers of internal groups of TFP-2 would strongly suppress the light intensity, while the instrumental contrast (related only to the Fabry-Pérot mirrors reflectance) would remain the same. This selectivity makes the instrument better suited for the study of biological samples, owing to the intrinsically lower sensitivity to depolarized elastic scattering.

We summarize the characteristics of the new setup as follows.

Contrast and luminosity.-The $150-\mathrm{dB}$ contrast documented in Fig. 1(c) is made possible not only by the six-pass geometry of the interferometer, but also by the adoption of multiple optical isolators inside the device [Fig. 1(b)], which prevent back propagation of light inside the interferometer. The unprecedented contrast reached by the TFP-2 HC is by far superior to that of previous $3+3$ pass tandem FabryPérot interferometers ( $100 \mathrm{~dB}$ for the TFP-1) [58]. In addition, the luminosity of our setup is $\sim 0.2$ which, together with the factor 5 improvement in the efficiency of the detector, opens up the possibility to map the viscoelastic properties of truly opaque or even reflecting samples.

Resolution.-The full width at half height of the elastic line [Fig. 1(c)] gives the spectral resolution, which is related to the FPs' mirror spacing and to their optical quality. With mirror spacing of $15 \mathrm{~mm}$ or higher, a value of linewidth better than $0.1 \mathrm{GHz}$ can be obtained. This is more than a factor 7 better than in VIPAs, enabling the study of acoustic attenuation and relaxation processes in viscoelastic media.

Acquisition time.-The high efficiency of the setup in collecting quasielastic scattered light, by using a polarizing cube and an edge filter as beam splitters and an avalanche photodiode as the photodetector, enables the acquisition time for a single spectrum to be reduced to seconds. It is thus possible to measure within a reasonable time both collective (by BLS) and single-particle (by RS) properties of materials that are heterogeneous in space (e.g., biological matter or geological samples) or changing over time (e.g., reactive or degradable systems). 
Spectral range.-The tandem configuration is very effective in suppressing (better than $40 \mathrm{~dB}$ ) the replicas of the transmission peaks of the two FP interferometers. By combining spectra recorded with mirror spacing in the range of $20-0.2 \mathrm{~mm}$, a spectral range from fractions of $\mathrm{GHz}$ to more than $1 \mathrm{THz}$ can be obtained. The spectral range is further extended to the high-frequency side by means of the Raman spectrometer, which can operate in the range 0.9-100 THz. The possibility of collecting a continuous spectrum in such a wide and informative range is not possible with other setups, even with the most recent Raman-Brillouin ones [27], due to the very limited spectral range accessible to VIPAs.

Analysis of Brillouin spectra.-Brillouin spectra presented in Figs. 2 and 3 originate from light inelastically scattered by thermally activated acoustic modes. In a backscattering experiment from ideal homogeneous and elastic solids, two single Brillouin peaks can be revealed at frequency shifts $\nu$ given by $\omega_{b}=2 \pi \nu= \pm V q$, where $V$ is the velocity of the longitudinal acoustic modes, $q=2 n k_{i}$ is the momentum exchanged in the scattering process, $n$ is the refractive index of the sample, and $k_{i}$ is the wavevector of incident light. In ideally elastic samples, the longitudinal elastic modulus $M$ is simply given by $M=\rho V^{2}$, where $\rho$ is the mass density of the sample. In the case of viscoelastic materials, damping mechanisms reduce the lifetime of acoustic modes, and the Brillouin spectrum can be described by a damped harmonic oscillator function:

$$
I(\omega)=\frac{I_{0}}{\pi} \cdot \frac{\omega_{b}^{2} \Gamma_{b}}{\left(\omega^{2}-\omega_{b}^{2}\right)^{2}+\omega^{2} \Gamma_{b}^{2}},
$$

where $\omega_{b}$ and $\Gamma_{b}$ approximately give the frequency position and the linewidth (FWHM) of Brillouin peaks. These parameters are related to the real $\left(M^{\prime}\right)$ and imaginary $\left(M^{\prime \prime}=\omega \eta_{L}\right)$ parts of the longitudinal elastic modulus through the relations:

$$
M^{\prime}=\rho \omega_{b}^{2} / q^{2} ; \quad \eta_{L}=\rho \Gamma_{b} / q^{2},
$$

Note that the elastic modulus can be expressed as $M^{\prime}=\rho(\lambda \nu / 2 n)^{2}$, where $\lambda$ is the wavelength of laser light. The simple hydrodynamic model described by Eq. (1), convoluted with the instrumental function, is appropriate to fit the whole Brillouin spectrum of materials (liquids or solids) with internal relaxation rates much faster than $\omega_{b}$. In the case of relaxations close to $\omega_{b}$, generalized hydrodynamic models can be used to model the spectrum, introducing a frequency dependence either of the modulus $M$ or, equivalently, of the longitudinal viscosity $\eta_{L}$ [59]. Luckily enough, also in this case Eq. (1) can be used to fit the spectrum in correspondence to the peak maximum and, through Eq. (2), to obtain the real and imaginary part of the modulus at the single frequency of the Brillouin peak [60]. This fast procedure is the most appropriate for mapping purposes. Conversely, long and accurate measurements would be needed to obtain the shape of the relaxation functions from the band shape of Brillouin spectra, extending far from the resonance [60].

Analysis of EDLS spectra.-The total EDLS spectrum reported in Fig. 4 is obtained by joining the experimental profiles collected by means of the interferometric and dispersive part of the setup. The interferometric portion of the spectrum $(1-2000 \mathrm{GHz})$ derives from the combination of different mirror separations, namely, $d=14,4,1$, 0.5 , and $0.2 \mathrm{~mm}$; for the latter distance, three FSRs are used in order to reach the thousands of $\mathrm{GHz}$ and the overlapping with the dispersive segment. The high-frequency part of the spectrum (from about $900 \mathrm{GHz}$ ) has been recorded by using a $1800-1 / \mathrm{mm}$ grating with an aperture slit of $300 \mu \mathrm{m}$ at the entrance of the monochromator. After the removal of the dark count contributions, low- and high-frequency portions are spliced, benefiting from a large overlapping. Further, the imaginary part of the dynamic susceptibility $\chi^{\prime \prime}(\nu)$ is calculated as the ratio between the intensity of depolarized light $I_{V H}(\nu)$ and $[n(\nu)+1]$, where $n(\nu)=$ $\exp \left[\left(h \nu / k_{B} T\right)-1\right]^{-1}$ is the Bose-Einstein occupation number.

Materials.-The Candida albicans CMC 1768 strain we use in this study is isolated from abdominal fluid of a patient in the Intensive Care Unit in Pisa Hospital. The identification is carried out by comparing the ITS and LSU D1/D2 sequence with GenBank [61] and specific databases $[62,63]$. Biofilms are obtained by submerging disks of aluminum foil (4-mm diameter) in Petri dishes containing YEPD medium (yeast extract $1 \%$, peptone $1 \%$, dextrose $2 \%$ from Difco Laboratories, Detroit, MI) inoculated with yeast cells at $\mathrm{OD}_{600}=0.2$. Petri dishes are placed in a rocking incubator at $37^{\circ} \mathrm{C}$ for $72 \mathrm{~h}$, then samples are washed twice with distilled sterile water and dried at room temperature for 1 week prior to analysis.

The epoxy-amine mixture we use in this study is made of diglycidyl ether of bisphenol A (DGEBA, molecular weight 348) and diethylenetriamine (DETA, purity $\geq 99 \%$ ) in the 5:2 stoichiometric ratio. The two monomer types are mutually reactive and polymerize by stepwise addition of the amino hydrogen to the epoxy group, with a rate of reaction strongly controlled by the temperature. The mixture is prepared by mixing for $2 \mathrm{~min}$ at room temperature and then it is transferred into the measurement cell, keeping it at a fixed $T=65^{\circ} \mathrm{C}$. Because of the presence of a multifunctional reagent, the reaction of DGEBA with DETA grows branched molecules and finally yields a network-polymer glass. Before reaching the vitrification point, the system goes through the gel point, where a percolating network of bonded particles has developed. The extent of reaction at the gel point, according to the classical theory of FloryStockmayer [42], is $\alpha_{\text {gel }}=0.50$ for our system.

The NIH/3T3 murine fibroblast cell line was purchased from American Type Culture Collection (ATCC). Cells are 
grown in Dulbecco Modified Eagle's Medium (DMEM) containing $10 \%(\mathrm{v} / \mathrm{v})$ heat-inactivated fetal bovine serum (FBS), $100 \mathrm{u} / \mathrm{ml}$ penicillin, $100 \mathrm{u} / \mathrm{ml}$ streptomycin (SIGMA Aldrich, St. Louis, MO) and maintained at $37^{\circ} \mathrm{C}$ in a $5 \% \mathrm{CO}$ humidified atmosphere. Cells are seeded onto $\mathrm{CaF}_{2}$ substrates sterilized using $100 \%$ ethanol and $\mathrm{UV}$ irradiation, treated with poly-L-lysine solution. Fixation is performed by incubating cells with $4 \%$ paraformaldehyde in phosphate buffer saline (PBS) solution for $10 \mathrm{~min}$ at room temperature, then cells are washed twice with PBS.

\section{ACKNOWLEDGMENTS}

S. Corezzi acknowledges financial support from MIURPRIN (Project No. 2012J8X57P). S. Caponi acknowledges support from PAT (Provincia Autonoma di Trento) (GP/ PAT/2012) "Grandi Progetti 2012" Project "MaDEleNA." P.S., A. M., M.P. acknowledge financial support from Centro Nazionale Trapianti (Project: "Studio di cellule per uso clinico umano, con particolare riferimento a modelli cellulari (liposomi) e linee cellulari in interazione con crioconservanti e con materiali biocompatibili"). L. C. and S. Caponi acknowledge financial support from Consiglio Nazionale delle Ricerche-Istituto Officina dei Materiali. F. P. acnowledges support from the UK Engineering and Physical Sciences Research Council (Grant No. EP/M028739/1 (F. P.)). The authors acknowledge Jacopo Scarponi for valuable help in setting up the hardware and software system for simultaneous Raman and BLS measurements.

[1] L. Brillouin, Diffusion de la Lumiere et des Rayonnes X par un Corps Transparent Homogene; Influence del'Agitation Thermique., Ann. Phys. (Paris) 9, 88 (1922).

[2] C. V. Raman and K. S. Krishnan, The Optical Analogue of the Compton Effect, Nature (London) 121, 711 (1928).

[3] K. J. Koski, Non-Invasive Determination of the Complete Elastic Moduli of Spider Silks, Nat. Mater. 12, 262 (2013).

[4] D. Schneider et al., Nonlinear Control of High-Frequency Phonons in Spider Silk, Nat. Mater. 15, 1079 (2016).

[5] L. Comez, C. Masciovecchio, G. Monaco, and D. Fioretto, in Solid State Physics, 1st ed. (Elsevier, New York, 2012), Vol. 63, pp. 1-77.

[6] S. Tacchi et al., Universal Dependence of the Spin Wave Band Structure on the Geometrical Characteristics of TwoDimensional Magnonic Crystals, Sci. Rep. 5, 10367 (2015).

[7] M. Madami, S. Bonetti, G. Consolo, S. Tacchi, G. Carlotti, G. Gubbiotti, F. B. Mancoff, M. A. Yar, and J. Åkerman, Direct Observation of a Propagating Spin Wave Induced by Spin-Transfer Torque, Nat. Nanotechnol. 6, 635 (2011).

[8] J.-C. Beugnot, S. Lebrun, G. Pauliat, H. Maillotte, V. Laude, and T. Sylvestre, Brillouin Light Scattering from Surface Acoustic Waves in a Subwavelength-Diameter Optical Fibre, Nat. Commun. 5, 5242 (2014).
[9] T. Still, M. Mattarelli, D. Kiefer, G. Fytas, and M. Montagna, Eigenvibrations of Submicrometer Colloidal Spheres, J. Phys. Chem. Lett. 1, 2440 (2010).

[10] D. Schneider, P. J. Beltramo, M. Mattarelli, P. Pfleiderer, J. Vermant, D. Crespy, M. Montagna, E. M. Furst, and G. Fytas, Elongated Polystyrene Spheres as Resonant Building Blocks in Anisotropic Colloidal Crystals, Soft Matter 9, 9129 (2013).

[11] W. Cheng, J. Wang, U. Jonas, G. Fytas, and N. Stefanou, Observation and Tuning of Hypersonic Bandgaps in Colloidal Crystals, Nat. Mater. 5, 830 (2006).

[12] J. M. Vaughan and J.T. Randall, Brillouin Scattering, Density and Elastic Properties of the Lens and Cornea of the Eye, Nature (London) 284, 489 (1980).

[13] F. Palombo, C. P. Winlove, R. S. Edginton, E. Green, N. Stone, S. Caponi, M. Madami, and D. Fioretto, Biomechanics of Fibrous Proteins of the Extracellular Matrix Studied by Brillouin Scattering, J. R. Soc. Interface 11, 20140739 (2014).

[14] G. Antonacci , R. M. Pedrigi, A. Kondiboyina, V. V. Mehta, R. de Silva, C. Paterson, R. Krams, and P. Török Quantification of Plaque Stiffness by Brillouin Microscopy in Experimental Thin Cap Fibroatheroma, J. R. Soc. Interface 12, 20150843 (2015).

[15] G. Scarcelli, W. J. Polacheck, H. T. Nia, K. Patel, A. J. Grodzinsky, R. D. Kamm, and S. H. Yun, Noncontact Three-Dimensional Mapping of Intracellular Hydromechanical Properties by Brillouin Microscopy, Nat. Methods 12, 1132 (2015).

[16] M. Kawabe, K. Fukui, M. Matsukawa, M. Granke, A. Saïed, Q. Grimal, and P. Laugier, Comparative Investigation of Elastic Properties in a Trabecula Using Micro-Brillouin Scattering and Scanning Acoustic Microscopy, J. Acoust. Soc. Am. 132, EL54 (2012).

[17] I. R. Lewis and H. G. M. Edwards, Handbook of Raman Spectroscopy: From the Research Laboratory to the Process Line (Marcel Dekker, New York, 2001).

[18] S. Perticaroli, L. Comez, M. Paolantoni, P. Sassi, A. Morresi, and D. Fioretto, Extended Frequency Range Depolarized Light Scattering Study of N-Acetyl-leucine-methylamide-Water Solutions, J. Am. Chem. Soc. 133, 12063 (2011).

[19] L. Hong, B. Begen, A. Kisliuk, S. Pawlus, M. Paluch, and A. P. Sokolov, Influence of Pressure on Quasielastic Scattering in Glasses: Relationship to the Boson Peak, Phys. Rev. Lett. 102, 145502 (2009).

[20] N. J. Tao, G. Li, X. Chen, W. M. Du, and H. Z. Cummins, Low-Frequency Raman-Scattering Study of the LiquidGlass Transition in Aqueous Lithium Chloride Solutions, Phys. Rev. A 44, 6665 (1991).

[21] F. Palombo, M. Madami, N. Stone, and D. Fioretto, Mechanical Mapping with Chemical Specificity by Confocal Brillouin and Raman Microscopy, Analyst 139, 729 (2014).

[22] F. Palombo, M. Madami, D. Fioretto, J. Nallala, H. Barr, A. David, and N. Stone Chemico-Mechanical Imaging of Barrett's Oesophagus, J. Biophoton. 9, 694 (2016).

[23] G. Scarcelli and S. H. Yun, Confocal Brillouin Microscopy for Three-Dimensional Mechanical Imaging, Nat. Photonics 2, 39 (2008). 
[24] G. Scarcelli and S.H. Yun, Multistage VIPA Etalons for High-Extinction Parallel Brillouin Spectroscopy, Opt. Express 19, 10913 (2011).

[25] A. Fiore, J. Zhang, P. Shao, S. H. Yun, and G. Scarcelli, High-Extinction Virtually Imaged Phased Array-Vased Brillouin Spectroscopy of Turbid Biological Media, Appl. Phys. Lett. 108, 203701 (2016).

[26] Z. Meng, A. J. Traverso, and V. V. Yakovlev, Background Clean-up in Brillouin Microspectroscopy of Scattering Medium, Opt. Express 22, 5410 (2014).

[27] A. J. Traverso, J. V. Thompson, Z. A. Steelman, Z. Meng, M. O. Scully, and V. V. Yakovlev Dual Raman-Brillouin Microscope for Chemical and Mechanical Characterization and Imaging, Anal. Chem. 87, 7519 (2015).

[28] Z. Meng, S. C. Bustamante Lopez, K. E. Meissner, and V. V. Yakovlev, Subcellular Measurements of Mechanical and Chemical Properties Using Dual Raman-Brillouin Microspectroscopy, J. Biophoton. 9, 201 (2016).

[29] S. Y.-E. Tan, S. C. Chew, S. Y.-Y. Tan, M. Givskov, and L. Yang, Emerging Frontiers in Detection and Control of Bacterial Biofilms, Curr. Opin. Biotechnol. 26, 1 (2014).

[30] C. E. Zobell, The Effect of Solid Surfaces upon Bacterial Activity, J. Bacteriol. 46, 39 (1943).

[31] Y. A. Nikolaev and V. K. Plakunov, Biofilm-"City of Microbes" or an Analogue of Multicellular Organisms?, Microbiology 76, 125 (2007).

[32] K. Drescher, Y. Shen, B. L. Bassler, and H. A. Stone, Biofilm Streamers Cause Catastrophic Disruption of Flow with Consequences for Environmental and Medical Systems, Proc. Natl. Acad. Sci. U.S.A. 110, 4345 (2013).

[33] L. Corte et al., Exploring Ecological Modelling to Investigate Factors Governing the Colonization Success in Nosocomial Environment of Candida Albicans and Other Pathogenic Yeasts, Sci. Rep. 6, 26860 (2016).

[34] M. Trejo, C. Douarche, V. Bailleux, C. Poulard, S. Mariot, C. Regeard, and E. Raspaud, Elasticity and Wrinkled Morphology of Bacillus subtilis Pellicles, Proc. Natl. Acad. Sci. U.S.A. 110, 2011 (2013).

[35] R. Janissen, D. M. Murillo, B. Niza, P. K. Sahoo, M. M. Nobrega, C. L. Cesar, M. L. A. Temperini, H. F. Carvalho, A. A. de Souza, and M. A. Cotta, Spatiotemporal Distribution of Different Extracellular Polymeric Substances and Filamentation Mediate Xylella fastidiosa Adhesion and Biofilm Formation, Sci. Rep. 5, 9856 (2015).

[36] M. Okada, N. I. Smith, A. F. Palonpon, H. Endo, S. Kawata, M. Sodeoka, and K. Fujita, Label-Free Raman Observation of Cytochrome c Dynamics during Apoptosis, Proc. Natl. Acad. Sci. U.S.A. 109, 28 (2012).

[37] S. Caponi et al., Raman Micro-Spectroscopy Study of Living SH-SY5Y Cells Adhering on Different Substrates, Biophys. Chem. 208, 48 (2016).

[38] G. Scarcelli, P. Kim, and S. H. Yun, In Vivo Measurement of Age-Related Stiffening in the Crystalline Lens by Brillouin Optical Microscopy, Biophys. J. 101, 1539 (2011).

[39] K. Ioannidou, M. Kanduč, L. Li, D. Frenkel, J. Dobnikar, and E. D. Gado, The Crucial Effect of Early-Stage Gelation on the Mechanical Properties of Cement Hydrates, Nat. Commun. 7, 12106 (2016).

[40] J. Suhr, N. Koratkar, P. Keblinski, and P. Ajayan, Viscoelasticity in Carbon Nanotube Composites, Nat. Mater. 4, 134 (2005).
[41] R. Hardis, J. L. P. Jessop, F. E. Peters, and M. R. Kessler, Cure Kinetics Characterization and Monitoring of an Epoxy Resin Using DSC, Raman Spectroscopy, and DEA, Composites, Part A 49, 100 (2013).

[42] W. H. Stockmayer, Molecular Distribution in Condensation Polymers, J. Polym. Sci. A 9, 69 (1952).

[43] S. Corezzi, D. Fioretto, and P. Rolla, Bond-Controlled Configurational Entropy Reduction in Chemical Vitrification, Nature (London) 420, 653 (2002).

[44] B. Chu, G. Fytas, and G. Zalczer, Study of Thermal Polymerization of Styrene by Raman Scattering, Macromolecules 14, 395 (1981).

[45] S. Kondo, T. Igarashi, and T. Nakamura, Rayleigh-Brillouin Scattering from Reacting Epoxy Resins: Comparison with Torsional Braid Analysis, J. Appl. Polym. Sci. 26, 2337 (1981).

[46] I. Alig, D. Lellinger, K. Nancke, A. Rizos, and G. Fytas, Dynamic Light Scattering and Ultrasonic Investigations during the Cure Reaction of an Epoxy Resin, J. Appl. Polym. Sci. 44, 829 (1992).

[47] L. Comez, L. Lupi, A. Morresi, M. Paolantoni, P. Sassi, and D. Fioretto, More Is Different: Experimental Results on the Effect of Biomolecules on the Dynamics of Hydration Water, J. Phys. Chem. Lett. 4, 1188 (2013).

[48] S. Caponi, A. Fontana, F. Rossi, G. Baldi, and E. Fabiani, Effect of Temperature on the Vibrational Density of States in Vitreous $\mathrm{SiO}_{2}$ : A Raman Study, Phys. Rev. B 76, 092201 (2007).

[49] S. Caponi, S. Corezzi, D. Fioretto, A. Fontana, G. Monaco, and F. Rossi, Raman-Scattering Measurements of the Vibrational Density of States of a Reactive Mixture During Polymerization: Effect on the Boson Peak, Phys. Rev. Lett. 102, 027402 (2009).

[50] S. Perticaroli, L. Comez, M. Paolantoni, P. Sassi, L. Lupi, D. Fioretto, A. Paciaroni, and A. Morresi, Broadband Depolarized Light Scattering Study of Diluted Protein Aqueous Solutions, J. Phys. Chem. B 114, 8262 (2010).

[51] P. C. Painter, L. Mosher, and C. Rhoads, Low-Frequency Modes in the Raman Spectrum of DNA, Biopolymers 20, 243 (1981).

[52] T. S. Grigera, V. Martín-Mayor, G. Parisi, and P. Verrocchio, Phonon Interpretation of the "Boson Peak" in Supercooled Liquids, Nature (London) 422, 289 (2003).

[53] S. Perticaroli et al., Painting Biological Low-Frequency Vibrational Modes from Small Peptides to Proteins, Phys. Chem. Chem. Phys. 17, 11423 (2015).

[54] S. Khodadadi and A. P. Sokolov, Protein Dynamics: From Rattling in a Cage to Structural Relaxation, Soft Matter 11, 4984 (2015).

[55] D. A. Turton, H. M. Senn, T. Harwood, A. J. Lapthorn, E. M. Ellis, and K. Wynne Terahertz Underdamped Vibrational Motion Governs Protein-Ligand Binding in Solution, Nat. Commun. 5, 3999 (2014).

[56] S. Perticaroli, J. D. Nickels, G. Ehlers, and A. P. Sokolov, Rigidity, Secondary Structure, and the Universality of the Boson Peak in Proteins, Biophys. J. 106, 2667 (2014).

[57] K.-C. Chou and B. Mao, Collective Motion in DNA and Its Role in Drug Intercalation, Biopolymers 27, 1795 (1988). 
[58] F. Nizzoli and J. R. Sandercock, Dynamical Properties of Solids (North-Holland, Amsterdam, 1990), Vol. 6, pp. 281-335.

[59] N. J. Tao, G. Li, and H. Z. Cummins, Brillouin-Scattering Study of the Liquid-Glass Transition in $\mathrm{CaKNO}_{3}$ : ModeCoupling Analysis, Phys. Rev. B 45, 686 (1992).

[60] D. Fioretto, L. Comez, G. Socino, L. Verdini, S. Corezzi, and P. A.Rolla, Dynamics of Density Fluctuations of a Glass-Forming Epoxy Resin Revealed by Brillouin Light Scattering, Phys. Rev. E 59, 1899 (1999).
[61] http://blast.ncbi.nlm.nih.gov/Blast.cgi.

[62] L. Irinyi et al., International Society of Human and Animal Mycology (ISHAM)-ITS Reference DNA Barcoding Database-The Quality Controlled Standard Tool for Routine Identification of Human and Animal Pathogenic Fungi, Med. Mycol. 53, 313 (2015).

[63] C. L. Schoch et al., Finding Needles in Haystacks: Linking Scientific Names, Reference Specimens and Molecular Data for Fungi, Oxford Database (University of Oxford, Oxford, 2014), DOI:10.1093/database/bau061. 